\title{
Transition-Metal-Ethylene Complexes as High-Capacity Hydrogen-Storage Media
}

\author{
E. Durgun, ${ }^{1,2}$ S. Ciraci, ${ }^{1,2, *}$ W. Zhou, ${ }^{3,4}$ and T. Yildirim ${ }^{3,4}$ \\ ${ }^{1}$ Department of Physics, Bilkent University, Ankara 06800, Turkey \\ ${ }^{2}$ UNAM-National Nanotechnology Research Center, Bilkent University, Ankara 06800, Turkey \\ ${ }^{3}$ NIST Center for Neutron Research, National Institute of Standards and Technology, Gaithersburg, Maryland 20899, USA \\ ${ }^{4}$ Department of Materials Science and Engineering, University of Pennsylvania, Philadelphia, Pennsylvania 19104, USA
}

(Received 1 September 2006; published 30 November 2006)

\begin{abstract}
From first-principles calculations, we predict that a single ethylene molecule can form a stable complex with two transition metals (TM) such as Ti. The resulting TM-ethylene complex then absorbs up to ten hydrogen molecules, reaching to gravimetric storage capacity of $\sim 14 \mathrm{wt} \%$. Dimerization, polymerizations, and incorporation of the TM-ethylene complexes in nanoporous carbon materials are also discussed. Our results are quite remarkable and open a new approach to high-capacity hydrogen-storage materials discovery.
\end{abstract}

DOI: 10.1103/PhysRevLett.97.226102

Hydrogen is considered to be one of the best alternative and renewable fuels $[1,2]$ because of its abundance, easy synthesis, and nonpolluting nature when used in fuel cells. However, the main concern is the safe storage and efficient transport of this highly flammable gas [3].

The main obstacles in hydrogen storage are slow kinetics, poor reversibility and high dehydrogenation temperatures for the chemical hydrides [4], and very low desorption temperatures or energies for the physisorption materials [metal-organic frameworks (MOF) [5], carbidederived carbons [6], etc.]. Recently, a novel concept to overcome these obstacles has been suggested [7-14]. It was predicted that a single $\mathrm{Ti}$ atom affixed to carbon nanostructures, such as $\mathrm{C}_{60}$ or nanotubes, strongly adsorbs up to four hydrogen molecules $[7,8,10]$. The interaction between hydrogen molecules and transition metals is very unique, lying between chemi- and physisorption, with a binding energy of $0.4 \mathrm{eV}$ compatible with room temperature desorption/absorption. The origin of this unusual "molecular chemisorption" is explained by well-known Dewar coordination and Kubas interaction [15]. The transition metals (TM) are chemically bonded onto different molecules or nanostructures through hybridization of lowest-unoccupied molecular orbital (LUMO) of nanostructure with TM $d$-orbitals (i.e., Dewar coordination). The resulting complex then binds multiple molecular hydrogens through hybridization between $\mathrm{H}_{2}-\sigma^{*}$ antibonding and TM $d$ orbitals (i.e., Kubas interactions).

Synthesizing the predicted structures of $\mathrm{Ti}$ decorated nanotubes $/ \mathrm{C}_{60}$ was proven to be very difficult because of the lack of bulk quantities of small-diameter nanotubes and strong $\mathrm{C}_{60}-\mathrm{C}_{60}$ interactions in the solid phase. Moreover, $\mathrm{Ti}$ atoms uniformly coating the $\mathrm{SWNT} / \mathrm{C}_{60}$ surface may be subject to clustering after several charging-discharging processes [16].

In searching for a more efficient and feasible highcapacity hydrogen-storage medium, we found that the $\mathrm{C}=$ $\mathrm{C}$ double bond of an ethylene molecule $\mathrm{C}_{2} \mathrm{H}_{4}$, mimics double bonds of $\mathrm{C}_{60}$ that strongly binds the TM atom
PACS numbers: 68.43.Bc, 81.07.-b, 84.60.Ve

(see Fig. 1) and therefore it is expected to support TM atoms strongly to provide a basis for high-capacity hydrogen storage via the Dewar-Kubas mechanism discussed above.

In this Letter, we explored this idea and indeed found that a single ethylene molecule can hold not only one but also two $\mathrm{Ti}$ atoms, i.e., $\mathrm{C}_{2} \mathrm{H}_{4} \mathrm{Ti}_{2}$, which then reversibly binds up to ten $\mathrm{H}_{2}$ molecules yielding an unexpectedly high storage capacity of $\sim 14 \mathrm{wt} \%$. These results suggest that ethylene, a well-known inexpensive molecule, can be an important basis in developing frameworks for efficient and safe hydrogen-storage media.

Our results are obtained from first-principles plane wave calculations within density functional theory using Vanderbilt-type ultrasoft pseudopotentials with PerdewZunger exchange correlation [17]. Single molecules have been treated in a supercell of $15 \times 15 \times 15 \AA$ with $\Gamma$

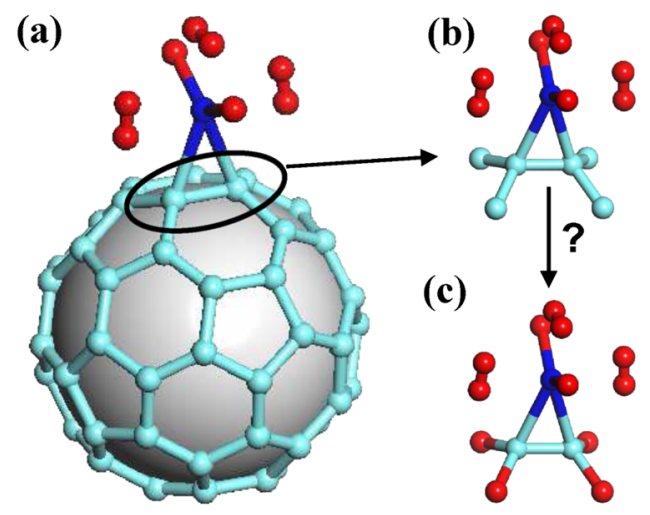

FIG. 1 (color online). (a) One of the most stable structures of the $\mathrm{Ti}^{-} \mathrm{C}_{60}$ complex where the $\mathrm{Ti}$ atom (black) is bonded to a double bond with four hydrogen molecules attached (dark gray). (b) The local structure of the Ti- $\mathrm{C}_{60}$ double bond. (c) Replacing the end carbon atoms shown in (b) by $\mathrm{H}$ results in an ethylene molecule. This suggests that we may simply use the ethylene molecule to hold Ti atoms, which then binds multiple hydrogen molecules. 
$k$-point and a cutoff energy of $408 \mathrm{eV}$. The structures are optimized until the maximum force allowed on each atom is less than $0.01 \mathrm{eV} / \AA$ for both spin-paired and spinrelaxed cases.

We first studied the bonding of a single Ti-atom to an ethylene molecule to form $\mathrm{C}_{2} \mathrm{H}_{4} \mathrm{Ti}$ [see Fig. 2(b)]. We found no energy barrier for this reaction. The binding energy is calculated by subtracting the equilibrium total energy $E_{T}$ of $\mathrm{C}_{2} \mathrm{H}_{4} \mathrm{Ti}$ molecule from the sum of the total energies of free ethylene molecule and of Ti-atom; $E_{B}(\mathrm{Ti})=E_{T}\left(\mathrm{C}_{2} \mathrm{H}_{4}\right)+E_{T}(\mathrm{Ti})-E_{T}\left(\mathrm{C}_{2} \mathrm{H}_{4} \mathrm{Ti}\right)$. The $\mathrm{Ti}$ atom forms a symmetric bridge bond with the $\mathrm{C}=\mathrm{C}$ bond of ethylene with $E_{B}=1.45 \mathrm{eV}$. Interestingly, it is also possible to attach a second $\mathrm{Ti}$ atom to the $\mathrm{C}_{2} \mathrm{H}_{4} \mathrm{Ti}$ to form $\mathrm{C}_{2} \mathrm{H}_{4} \mathrm{Ti}_{2}$ [see Fig. 2(c)] without any potential barrier and about the same binding energy as the first Ti atom. In the optimized structure [Fig. 2(c)], each Ti atom is closer to one of the carbon atoms, leading to two different Ti-C bonds. Figure 2(d) shows that the bonding orbital for the Ti atoms and $\mathrm{C}_{2} \mathrm{H}_{4}$ results from the hybridization of the LUMO orbital of the ethylene molecule and the Ti- $d$ orbitals, in accord with Dewar coordination [15]. The spin-polarized calculation gives $1.53 \mathrm{eV}$ lower energy than the non-spin-polarized one, suggesting a magnetic ground state for $\mathrm{C}_{2} \mathrm{H}_{4} \mathrm{Ti}_{2}$ with moment $\mu=6 \mu_{B}$ per molecule.

The stability of the $\mathrm{C}_{2} \mathrm{H}_{4} \mathrm{Ti}_{2}$ complex was further tested by normal mode analysis. We found no soft (i.e., negative) mode. There are three main Ti modes. In two of these modes, $\mathrm{Ti}$ atoms vibrate parallel and perpendicular to the $\mathrm{C}=\mathrm{C}$ bond and have the energies of 176 and $123 \mathrm{~cm}^{-1}$, respectively. In the third $\mathrm{Ti}$ mode, $\mathrm{Ti}$ atoms vibrate perpendicular to the $\mathrm{C}_{2} \mathrm{H}_{4}$ plane with an energy of $367 \mathrm{~cm}^{-1}$. These three modes are unique for the $\mathrm{C}_{2} \mathrm{H}_{4} \mathrm{Ti}_{2}$ complex (a)

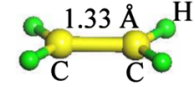

(b)
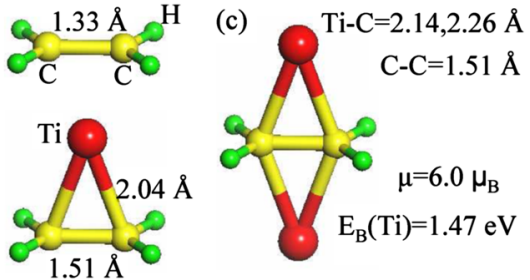

(d)

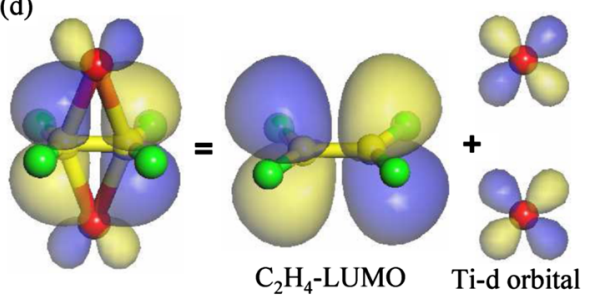

FIG. 2 (color online). Optimized structure of an ethylene molecule $\mathrm{C}_{2} \mathrm{H}_{4}$ (a), $\mathrm{C}_{2} \mathrm{H}_{4} \mathrm{Ti}$ (b), and $\mathrm{C}_{2} \mathrm{H}_{4} \mathrm{Ti}_{2}$ (c). The panel (d) shows that the $\mathrm{Ti}-\mathrm{C}_{2} \mathrm{H}_{4}$ bonding orbital results from the hybridization of the LUMO of the $\mathrm{C}_{2} \mathrm{H}_{4}$ and the Ti- $d$ orbital, in accord with Dewar coordination. and therefore should be present in any Raman/IR spectra of a successfully synthesized material.

We next studied the $\mathrm{H}_{2}$ storage capacity of the Tiethylene complex by calculating the interaction between $\mathrm{C}_{2} \mathrm{H}_{4} \mathrm{Ti}_{2}$ and a different number of $\mathrm{H}_{2}$ molecules and configurations. The first $\mathrm{H}_{2}$ molecule is absorbed dissociatively to form $\mathrm{C}_{2} \mathrm{H}_{4}\left(\mathrm{TiH}_{2}\right)_{2}$ as shown in Fig. 3(a) with a binding energy of $1.18 \mathrm{eV} / \mathrm{H}_{2}$. The additional hydrogen molecules do not dissociate and molecularly absorbed around the Ti atom. Two of these configurations are shown in Figs. 3(b) and 3(c). In the $\mathrm{C}_{2} \mathrm{H}_{4}\left(\mathrm{TiH}_{2}-2 \mathrm{H}_{2}\right)_{2}$ configuration, two $\mathrm{H}_{2}$ are molecularly bonded from the left and right side of the $\mathrm{TiH}_{2}$ group with a binding energy of $0.38 \mathrm{eV} / \mathrm{H}_{2}$ and significantly elongated $\mathrm{H}-\mathrm{H}$ bond length of $0.81 \AA$. It is also energetically favorable to add a third $\mathrm{H}_{2}$ molecule from the top of the $\mathrm{TiH}_{2}$ group, with a binding energy of $0.4 \mathrm{eV}$ and bond length of $0.82 \AA$. The resulting structure, $\mathrm{C}_{2} \mathrm{H}_{4}\left(\mathrm{TiH}_{2}-3 \mathrm{H}_{2}\right)_{2}$, is shown in Fig. 3(c). We note that these binding energies have the right order of magnitude for room temperature storage. Since the hydrogens are absorbed molecularly, we also expect fast absorption or desorption kinetics.

Finally, we also observed many other local stable configurations where all of the hydrogen molecules are bonded molecularly. One such configuration, denoted as $\mathrm{C}_{2} \mathrm{H}_{4}\left(\mathrm{Ti}-5 \mathrm{H}_{2}\right)_{2}$, is shown in Fig. 3(d). Here the $\mathrm{H}_{2}$ molecules stay intact and benefit equally from bonding with the Ti atom. The total ten hydrogen molecules absorbed by a

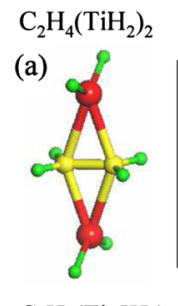

$\mathrm{C}_{2} \mathrm{H}_{4}\left(\mathrm{TiH}_{2}-2 \mathrm{H}_{2}\right)_{2}$ (b)
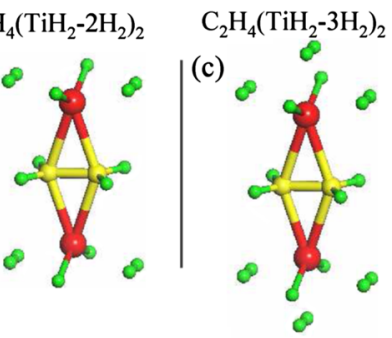

(d)

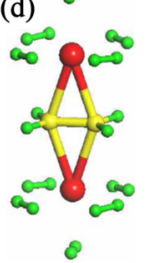

(e)
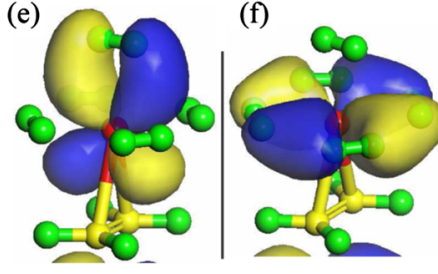

FIG. 3 (color online). Atomic configurations of an ethylene molecule functionalized by two Ti atoms, holding (a) two $\mathrm{H}_{2}$ molecules which are dissociated, (b) six $\mathrm{H}_{2}$ molecules, and (c) eight $\mathrm{H}_{2}$ molecules. Panel (d) shows a configuration where ten $\mathrm{H}_{2}$ are bonded all molecularly. The spin-polarized calculations gave lower energies (in eV) by 1.5, 0.37, 0.16, and 0.06 for configurations shown in (a)-(d), respectively, suggesting a magnetic ground state for all cases with a moment of $\mu \approx 2 \mu_{B}$. Panels (e) and (f) show the bonding orbital for the top (e) and side hydrogen molecules, respectively. Note that the hydrogen $\sigma^{*}$-antibonding orbitals are hybridized with Ti- $d$ orbitals, suggesting Kubas interaction for the $\mathrm{H}_{2}$-Ti bonding. 
single Ti-ethylene complex, $\mathrm{C}_{2} \mathrm{H}_{4}\left(\mathrm{Ti}-5 \mathrm{H}_{2}\right)_{2}$, correspond to $\mathrm{a} \sim 14 \mathrm{wt} \%$ gravimetric density, which is more than twice of the criterion set for efficient hydrogen storage. By removing the top $\mathrm{H}_{2}$ molecule, we find that $\mathrm{C}_{2} \mathrm{H}_{4}\left(\mathrm{Ti}-4 \mathrm{H}_{2}\right)_{2}$ configuration is also a local minimum and has a slightly $\left(0.08 \mathrm{eV} / \mathrm{H}_{2}\right)$ higher energy than the $\mathrm{C}_{2} \mathrm{H}_{4}\left(\mathrm{TiH}_{2}-3 \mathrm{H}_{2}\right)_{2}$ configuration shown in Fig. 3(c). Our MD simulations indicate that the system oscillates between these two configurations. This is consistent with the observation that in Kubas compounds [15], the dihydrogen (i.e., $2 \mathrm{H}$ ) and molecular (i.e., $\mathrm{H}_{2}$ ) bonding are usually found to be in resonance [15].

The top hydrogen molecule in $\mathrm{C}_{2} \mathrm{H}_{4}\left(\mathrm{Ti}-5 \mathrm{H}_{2}\right)_{2}$ has the weakest bonding in the system with $E_{B}=0.29 \mathrm{eV}$, whereas the side $\mathrm{H}_{2}$ molecules have the strongest bonding with $E_{B}=0.49 \mathrm{eV} / \mathrm{H}_{2}$ and significantly elongated $\mathrm{H}-\mathrm{H}$ bond distance of $0.85 \AA$. This suggests the presence of two different $\mathrm{H}_{2}-\mathrm{C}_{2} \mathrm{H}_{4} \mathrm{Ti}_{2}$ bonding orbitals as shown in Figs. 3(e) and 3(f). The first one is the hybridization between the top- $\mathrm{H}_{2} \sigma^{*}$-antibonding and the Ti- $d$ orbital. The second one is the simultaneous hybridization of the side $\mathrm{H}_{2} \sigma^{*}$-antibonding orbitals with the Ti- $d$ orbital. Since the bonding orbitals are mainly between metal $d$ - and hydrogen $\sigma^{*}$-antibonding orbitals, the mechanism of this interesting interaction can be explained by the Kubas interaction [15].

We also calculated the normal modes of $\mathrm{C}_{2} \mathrm{H}_{4}\left(\mathrm{Ti}-5 \mathrm{H}_{2}\right)_{2}$ and did not find any soft modes, indicating that the system indeed corresponds to a local minimum. Among many vibrational modes, we note that the $\mathrm{H}_{2}$ stretching mode is around $2700-3000 \mathrm{~cm}^{-1}$ for side $\mathrm{H}_{2}$ and around $3300 \mathrm{~cm}^{-1}$ for top $\mathrm{H}_{2}$ molecules, significantly lower than the $4400 \mathrm{~cm}^{-1}$ for free $\mathrm{H}_{2}$ molecule. Such a shift in the mode frequency would be the key feature that can be probed by Raman/IR measurement to confirm a successful synthesis of the structures predicted here.

Finally, the stability of $\mathrm{C}_{2} \mathrm{H}_{4}\left(\mathrm{Ti}-5 \mathrm{H}_{2}\right)_{2}$ structure has been further studied by carrying out $a b$ initio molecular dynamic calculations performed at 300 and $800 \mathrm{~K}$. While all ten $\mathrm{H}_{2}$ molecules have remained bound to the $\mathrm{C}_{2} \mathrm{H}_{4} \mathrm{Ti}_{2}$ molecule at $300 \mathrm{~K}$, they start to desorb above $300 \mathrm{~K}$ and all of them are desorbed already at $800 \mathrm{~K}$, leaving behind a stable $\mathrm{C}_{2} \mathrm{H}_{4} \mathrm{Ti}_{2}$ molecule. This prediction suggests that all the stored hydrogen molecules can be discharged easily through heating.

We next discuss the possibility of the dimerization and polymerization in the course of recycling and its effect on the hydrogen storage capacity. We found that two molecules can form a dimer through a Ti-Ti bond as shown in Fig. 4(a). The dimer formation is exothermic with an energy gain of $E_{D}=2.28 \mathrm{eV}$, and leads to a stable structure. The ground state is ferromagnetic with $\mu=6 \mu_{B}$. While each $\mathrm{Ti}$ atom at both ends of the dimer can bind 5 $\mathrm{H}_{2}$ molecules, two linking Ti-atom can absorb only $4 \mathrm{H}_{2}$ totaling to $14 \mathrm{H}_{2}$ per dimer. As a result, the gravimetric density obtained by the dimer is lowered to $\sim 10 \mathrm{wt} \%$. The (a)
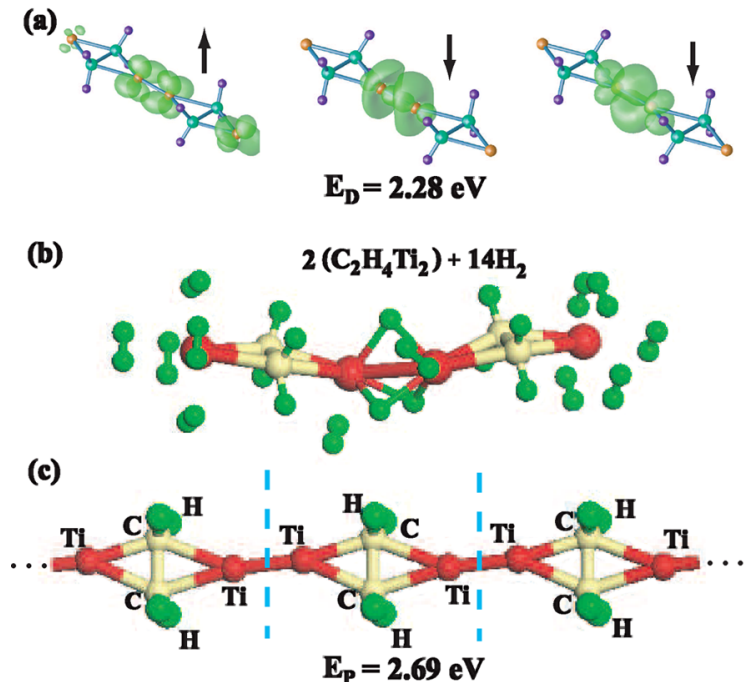

(d)

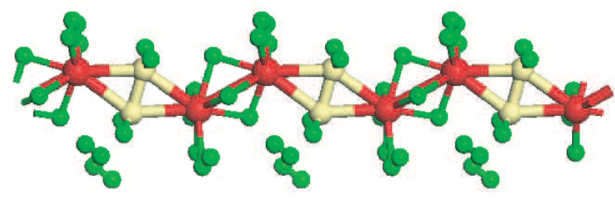

(e)

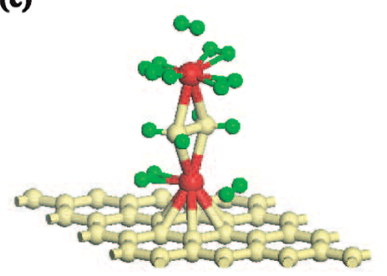

(I)

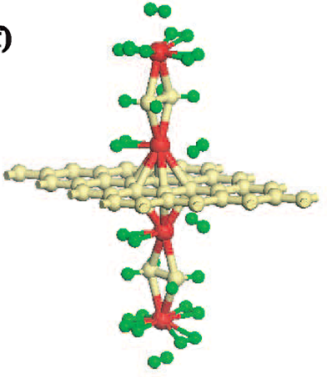

FIG. 4 (color online). (a) Atomic configuration and charge density plots of a dimer of $\mathrm{C}_{2} \mathrm{H}_{4} \mathrm{Ti}_{2}$ molecules linked by two $\mathrm{Ti}$ atoms one from either molecule. Large, medium, and small balls indicate titanium, carbon, and hydrogen atoms, respectively. Charge density plots (from left to right) correspond to HOMO spin-up, and two spin-down states. (b) Atomic configuration of $\mathrm{C}_{2} \mathrm{H}_{4} \mathrm{Ti}_{2}$ dimer holding $14 \mathrm{H}_{2}$ molecules. (c) Polymer of $\mathrm{C}_{2} \mathrm{H}_{4} \mathrm{Ti}_{2}$ molecule with polymerization energy $E_{P}$. (d) Atomic configuration of the polymer holding $6 \mathrm{H}_{2}$ molecule. (e) A $\mathrm{C}_{2} \mathrm{H}_{4} \mathrm{Ti}_{2}$ molecule adsorbed above the center of a hexagon in a $(3 \times 3)$ cell holding $7 \mathrm{H}_{2}$. (f) Two Ti-ethylene complexes adsorbed above and below the center of the same hexagon holding $14 \mathrm{H}_{2}$.

total energy can be further lowered by adding more $\mathrm{C}_{2} \mathrm{H}_{4} \mathrm{Ti}_{2}$ molecules to the dimer, and eventually by forming a paramagnetic polymer as shown in Fig. 4(c). The polymerization energy per molecule is calculated to be $E_{P}=2.69 \mathrm{eV}$ and the $\mathrm{H}_{2}$ storage capacity is further lowered to $6.1 \mathrm{wt} \%$. Polymerization did not change the binding energy of the $\mathrm{H}_{2}$ on Ti-atom significantly, and therefore should not affect the desorption temperature.

In order to prevent $\mathrm{C}_{2} \mathrm{H}_{4} \mathrm{Ti}_{2}$ molecules from forming a possible polymer phase during recycling, one can imagine 
TABLE I. The binding energies (in eV) with respect to atomic and bulk energies of various metals $(M$ ). The last two rows indicate the maximum number of $\mathrm{H}_{2}$ molecules bonded to each metal and its average binding energy (in $\mathrm{eV}$ ).

\begin{tabular}{|c|c|c|c|c|c|c|c|c|c|c|c|c|c|c|c|}
\hline Property/M & $\mathrm{Sc}$ & $\mathrm{Ti}$ & V & $\mathrm{Cr}$ & Mn & $\mathrm{Fe}$ & Co & $\mathrm{Ni}$ & $\mathrm{Cu}$ & $\mathrm{Zn}$ & $\mathrm{Zr}$ & Mo & $\mathrm{W}$ & $\mathrm{Pd}$ & $\mathrm{Pt}$ \\
\hline$E_{B}(M$-atomic $)$ & 1.39 & 1.47 & 1.27 & 0.05 & 0.37 & 0.83 & 1.30 & 0.70 & 1.41 & none & 1.69 & 0.37 & 1.18 & 1.56 & 1.78 \\
\hline$E_{B}(M$-bulk $)$ & -2.72 & -3.66 & -4.13 & -3.57 & -3.20 & -1.74 & -2.53 & -2.19 & -2.25 & - & -4.44 & -5.84 & -7.18 & -2.24 & -3.56 \\
\hline $\max \mathrm{H}_{2} / M$ & 5 & 5 & 5 & 5 & 5 & 5 & 3 & 2 & 2 & - & 5 & 5 & 5 & 2 & 2 \\
\hline$E_{B}\left(\right.$ per $\left.\mathrm{H}_{2}\right)$ & 0.39 & 0.45 & 0.43 & 0.35 & 0.34 & 0.26 & 0.41 & 0.87 & 0.14 & - & 0.57 & 0.77 & 0.90 & 0.58 & 0.95 \\
\hline
\end{tabular}

incorporating these TM-ethylene complexes in a nanoporous material such as MOF [5] and carbide-derived carbons [6]. As an example, we consider a $\mathrm{C}_{2} \mathrm{H}_{4} \mathrm{Ti}_{2}$ adsorbed above and below the center of a hexagon of a graphene layer. Here the graphene is taken as a prototype system which represents the internal structure of a carbon-based nanoporous material. We find that TM-ethylene complex can form a stable structure with the graphene surface. As shown in Figs. 4(e) and 4(f), single and double $\mathrm{C}_{2} \mathrm{H}_{4} \mathrm{Ti}_{2}$ molecules assembled on a $3 \times 3$ graphene layer can hold 7 and $14 \mathrm{H}_{2}$ with an average binding energy of 0.43 and $0.41 \mathrm{eV} / \mathrm{H}_{2}$, respectively. The binding energy of the $\mathrm{C}_{2} \mathrm{H}_{4} \mathrm{Ti}_{2}$ molecule is found to be $\sim 2 \mathrm{eV}$. The actual binding energy in nanoporous materials could be even higher because of curvature effects [18]. The maximum gravimetric density achieved in this present framework is $6.1 \mathrm{wt} \%$.

It is important to know if the results reported above for $M=$ Ti hold for other metals. Therefore we are currently studying a large number of metals and the details will be published elsewhere [19]. Our initial results are summarized in Table I, which clearly indicates that most of the light TM atoms can be bound to ethylene and each of them can absorb up to five $\mathrm{H}_{2}$ molecules. Scandium is the ideal case but for practical reasons $\mathrm{Ti}$ is the best choice of elements. $\mathrm{Cr}$ binds very weakly while $\mathrm{Zn}$ does not bind at all to the $\mathrm{C}_{2} \mathrm{H}_{4}$ molecule. Interestingly, $\mathrm{Zr}$ forms a stronger bonding with $\mathrm{C}_{2} \mathrm{H}_{4}$ than $\mathrm{Ti}$ and can absorb up to ten $\mathrm{H}_{2}$ molecularly with an average binding energy of $0.6 \mathrm{eV}$. Heavier metals such as Pd and Pt can also form complexes with $\mathrm{C}_{2} \mathrm{H}_{4}$ but bind fewer hydrogen molecules with significantly stronger binding energy than Ti. Table I also gives the binding energies with respect to bulk metal energies. The negative value for $E_{B}$ indicates endothermic reaction. Because of the very low vapor pressure of many metals, it is probably better to use some metal precursor to synthesize the structures predicted here.

In conclusion, we showed that an individual ethylene molecule functionalized by two light transition metals can bind up to ten hydrogen molecules via Dewar-Kubas interaction, reaching a gravimetric density as high as $\sim 14 \mathrm{wt} \%$. We propose to incorporate the TM-ethylene complex into carbon-based nanoporous materials to avoid dimerization/polymerization during recycling. Our results open a new approach to the high-capacity hydrogen- storage materials discovery by functionalizing small organic molecules with light transition metals.

This work was partially supported by TÜBİTAK under Grant No. TBAG-104T536. W.Z. and T. Y. acknowledge partial support from DOE under DE-FC36-04GO14282 and BES Grant No. DE-FG02-98ER45701. We thank Dr. Sefa Dag for fruitful discussions.

*Electronic address: ciraci@fen.bilkent.edu.tr

[1] See the special issue Towards a Hydrogen Economy, by R. Coontz and B. Hanson, Science 305, 957 (2004).

[2] G. W. Crabtree, M.S. Dresselhaus, and M. V. Buchanan, Phys. Today 57, No. 12, 39 (2004).

[3] A. Zuttel, Mater. Today 6, 24 (2003).

[4] B. Bogdanovic et al., Adv. Mater. 15, 1012 (2003).

[5] T. Yildirim and J. Hartman, Phys. Rev. Lett. 95, 215504 (2005).

[6] Y. Gogotsi et al., J. Am. Chem. Soc. 127, 16006 (2005).

[7] T. Yildirim and S. Ciraci, Phys. Rev. Lett. 94, 175501 (2005).

[8] T. Yildirim, J. Iniguez, and S. Ciraci, Phys. Rev. B 72, 153403 (2005).

[9] Y. Zhao et al., Phys. Rev. Lett. 94, 155504 (2005).

[10] S. Dag, Y. Ozturk, S. Ciraci, and T. Yildirim, Phys. Rev. B 72, 155404 (2005).

[11] Y. Zhao et al., Chem. Phys. Lett. 425, 273 (2006).

[12] N. Akman, E. Durgun, T. Yildirim, and S. Ciraci, J. Phys. Condens. Matter 18, 9509 (2006).

[13] B. Kiran, A. K. Kandalam, and P. Jena, J. Chem. Phys. 124, 224703 (2006).

[14] H. Lee, W. I. Choi, and J. Ihm, Phys. Rev. Lett. 97, 056104 (2006).

[15] Metal Dihydrogen and Bond Complexes-Structure, Theory and Reactivity, edited by G. J. Kubas (Kluwer Academic/ Plenum Publishing, New York, 2001).

[16] Q. Sun, Q. Wang, P. Jena, and Y. Kawazoe, J. Am. Chem. Soc. 127, 14582 (2005).

[17] S. Baroni, A. Dal Corso, S. de Gironcoli, and P. Giannozzi, http://www.pwscf.org. Part of the calculations have been confirmed by VASP software: G. Kresse and J. Hafner, Phys. Rev. B 47, 558 (1993).

[18] O. Gulseren, T. Yildirim, and S. Ciraci, Phys. Rev. Lett. 87, 116802 (2001).

[19] W. Zhou, T. Yildirim, E. Durgun, and S. Ciraci, Phys. Rev. $\mathrm{B}$ (to be published). 